\title{
METHOD FOR IDENTIFICATION AND FORECASTING THE STATE OF ECONOMIC DYNAMIC SYSTEMS
}

\section{Dymova H. O.}

\section{INTRODUCTION}

The analysis of economic processes involves two approaches fundamental and technical. The fundamental approach is to build a hypothesis about the possibility of the influence of a number of changed conditions on the economic indicator in the future. Knowing what significance the factor will take in the future, it is possible to predict this process. The technical approach is to study the change in indicator as a function of time. Here the hypothesis is accepted that the trends of the past will be repeated in the future. In this case, factors affecting the process is neglected.

In modern economic and analytical activities, dynamic models, which include both approaches to forecasting, are increasingly used. The development of economic policy at both the macro and micro levels requires solving problems that determine what impact the values of the controlled variables of the current period will have on the future values of economic indicators.

The process of identifying and forecasting information data in economics is that it is necessary to define a model and identify it with a library of known models or signals. The described task can present significant difficulties if information data is subjected to large distortion.

Information recovery problems for dynamic systems have been considered in many works and are represented by three tasks:

1) an identification task, when, on the basis of known signals at the input and output of the system, a conclusion is made about the composition of the system and its characteristics;

2) a control task when the characteristics of the system and the input signal are known and is determined the law of change of the signal at the output of the system or such an input signal that at the output brings the system to a given state;

3) measurement task, when the output signal and the characteristics of the system are known, the characteristics of the input signal are determined.

In many problems, there is no information about the input signal and the characteristics of the system, but the measured characteristics of the output process are known, therefore, it is necessary to study factorization algorithms. Regardless of any identification or assessment considerations, it is desirable 
to find a model to describe the dynamics of process generation. Thus, there is a problem of determining the characteristics of the system and describing the dynamics of the input process.

\section{Presentation of random processes in the state variables}

To develop a method for identifying and forecasting the state of dynamic systems in economics, it is necessary to introduce the concept of specifying a random process in the form of a system with a variable state, which can be used to generate a process.

A dynamical system that generates some random process in a linear representation can be specified by five matrices $\mathbf{A}(\mathrm{t}), \mathbf{B}(\mathrm{t}), \mathbf{C}(\mathrm{t}), \mathbf{Q}, \mathbf{P}_{i}$, a system of differential and algebraic equations, with given initial conditions:

$$
\begin{gathered}
\frac{d \vec{x}}{d t}=\mathbf{A}(t) \vec{x}(t)+\mathbf{B}(t) \vec{u}(t), \quad T_{i} \leq t \\
\vec{y}(t)=\mathbf{C}(t) \vec{x}(t), \quad T_{i} \leq t,
\end{gathered}
$$

where $\vec{x}(t)$ - state vector with the dimension $(n \times 1) ; \vec{u}(t)$ - white arousal process with the dimension $(p \times 1) ; \vec{y}(t)$ - observable process with the dimension $(m \times 1)$.

Equation (1) is a linear equation of state, and (2) is an observation equation. The matrices $\mathbf{A}(t), \mathbf{B}(t), \mathbf{C}(t)$ have dimensions $(n \times n),(n \times p)$, $(m \times n)$, respectively.

The input process $\vec{u}(t)$ has a covariance function of the form

$$
E\left[\vec{u}(t) \vec{u}^{T}(\tau)\right]=\mathbf{Q} \delta(t-\tau)
$$

The initial state vector is a random variable with a covariance matrix

$$
E\left[\vec{x}\left(T_{i}\right) \vec{x}^{T}\left(T_{i}\right)\right]=\mathbf{K}_{x}\left(T_{i}, T_{i}\right)=\mathbf{P}_{i},
$$

where $E$ - operator of mathematical expectation.

Many results of the theory of random processes can be expressed in terms of the matrix of covariance functions

$$
\mathbf{K}_{y}(t, \tau)=E\left[\vec{y}(t), \vec{y}^{T}(\tau)\right]
$$


To solve the problem of forecasting the state of dynamical systems it is necessary to create a signal library, that is the solution of the direct problem. Therefore, it is necessary to perform the search procedure for the covariance function of a random process by its representation in state variables.

\section{Determination of the covariance function of a random process by its description in state variables}

Definition of the covariance function of a random output process $\vec{y}(t)$ from its description in state variables is given according to ${ }^{1,2,3}$ without proof and used it to solve the inverse problem - finding the state variables from a known covariance matrix. According to $(2) \mathbf{K}_{y}(t, \tau)$ is easily related to the covariance matrix of the state vector $\overrightarrow{\mathbf{x}}(t)$ :

$$
\mathbf{K}_{y}(t, \tau)=\mathbf{C}(t) \mathbf{K}_{x}(t, \tau) \mathbf{C}^{T}(\tau)
$$

The covariance matrix $\mathbf{K}_{\mathrm{x}}(t, \tau)$, in its turn, satisfies the differential equation ${ }^{4}$

$$
\dot{\mathbf{K}}_{x}(t, \tau)=\mathbf{A}(t) \mathbf{K}_{x}(t, t)+\mathbf{K}_{x}(t, t) \mathbf{A}^{T}(t)+\mathbf{B}(t) \mathbf{Q} \mathbf{B}^{T}(t)
$$

at the initial condition $\mathbf{K}_{x}\left(T_{i}, T_{i}\right)=\mathbf{P}_{i}$.

Since $\vec{u}\left(t^{\prime}\right)$ and $\vec{x}(t)$ are not correlated on the integration interval of the differential equation (7), obtain

$$
\mathbf{K}_{\mathrm{x}}(t, \tau)=\left\{\begin{array}{l}
\Theta(t, \tau) \mathbf{K}_{x}(\tau, \tau), t \geq \tau \\
\mathbf{K}_{x}(t, t) \Theta^{T}(\tau, t), \tau \geq t
\end{array}\right.
$$

where $\Theta(t, \tau)$ - transition matrix obtained from the differential equation ${ }^{5}$ $\Theta\left(t, t_{0}\right)=\mathbf{A}(t) \Theta\left(t, t_{0}\right)$, at the initial condition $\Theta\left(t_{0}, t_{0}\right)=\mathbf{I}$, where $\mathbf{I}$ - identity matrix.

\footnotetext{
${ }^{1}$ Derusso, P., Roy, R., \& Klouz, C. (1970). Prostranstvo sostoyaniy v teorii upravleniya [State space in control theory]. Nauka.

${ }^{2}$ Van Trees, G.L. (1972). Teoriya obnaruzheniya, otsenok $i$ modulyatsii [The theory of detection, estimation and modulation] (T. 1). Sovetskoye radio.

${ }^{3}$ Kamke, E. (1971). Spravochnik po obyknovennym differentsial'nym uravneniyam [Handbook of ordinary differential equations]. Nauka.

${ }^{4}$ Van Trees, G.L. (1972). Teoriya obnaruzheniya, otsenok $i$ modulyatsii [The theory of detection, estimation and modulation] (T. 1). Sovetskoye radio.

${ }^{5}$ Baggeroer, A.B. (1968). State Variables, the Fredholm Theory, and Optimal Communications. M.I.T.
} 
The solution for the state vector $\vec{x}(t)$ at any moment of time has the form $\vec{x}(t)=\Theta\left(t, t_{0}\right) x\left(t_{0}\right)$. Property (8) is valid for all processes represented by state variables in the form (1) - (5). For the stationary case, if the parameters of the system generating the process $\vec{y}(t)$ are constant, then the transition matrix is determined by the exponential factor $\Theta(t, \tau)=e^{\mathbf{A}(t-\tau) 6}$.

In order for the matrix $\mathbf{K}_{x}(t, t+\Delta t)$ to be a function of only $\Delta t$, the matrix $\mathbf{K}_{x}(t, t)$ must, according to (8), be equated to a constant value $\mathbf{P}_{\infty}$. This constant matrix is a stationary solution to equation (7). So, it is possible to simulate segments of a stationary process using systems with constant parameters and setting the covariance matrix of the initial state $\mathbf{P}_{i}$ equal to $\mathbf{P}_{\infty}$. As shown in ${ }^{7}$, the stationary solution of equation (7) has the form

$$
\mathbf{P}_{\infty}=\int_{0}^{\infty} e^{\mathbf{A} t} \mathbf{B} \mathbf{Q} e^{\mathbf{A}^{T} t} d t=\frac{1}{2 \pi j} \int_{-j \infty}^{j \infty}[\mathbf{I} s-\mathbf{A}]^{-1} \mathbf{B} \mathbf{Q} \mathbf{B}^{\mathrm{T}}\left[-\mathbf{I} s-\mathbf{A}^{T}\right]^{-1} d s
$$

and the covariance matrix of the state vector

$$
\mathbf{K}_{x}(t, t+\Delta t)= \begin{cases}e^{-\mathbf{A} \Delta t} \mathbf{P}_{\infty}, & \Delta t \leq 0 \\ \mathbf{P}_{\infty} e^{\mathbf{A} \Delta t}, & \Delta t>0\end{cases}
$$

And hence, according to the formula (6)

$$
\mathbf{K}_{y}(t, t+\Delta t)=\left\{\begin{array}{l}
\mathbf{C}(t) e^{-\mathbf{A} \Delta t} \mathbf{P}_{\infty} \mathbf{C}^{T}(t), \Delta t \leq 0 \\
\mathbf{C}(t) \mathbf{P}_{\infty} e^{\mathbf{A}^{T} \Delta t} \mathbf{C}^{T}(t), \Delta t>0
\end{array},\right.
$$

that is, the correlation function of the system output is expressed in terms of the system state variables. Consequently, on the contrary, there should be an inverse problem: knowing the correlation matrix of the output for given $\mathbf{C}(t)$ and $\mathbf{P}_{\infty}=$ const, determine the structure of the transfer function of the system, that is, solve the problem of partial identification and forecasting of the economic system. To solve this problem, it is advisable to use the method of factorization of covariance functions.

${ }^{6}$ Van Trees, G. L. (1975). Teoriya obnaruzheniya, otsenok i modulyatsii [The theory of detection, estimation and modulation] (T. 2). Sovetskoye radio.

${ }^{7}$ Baggeroer, A.B. (1968). State Variables, the Fredholm Theory, and Optimal Communications. M.I.T. 


\section{Improvement of the factorization method of covariance functions}

Previously, it was assumed that there is a description of a random process in state variables, and a model for determining the covariance function (matrix) of this process was proposed. Now consider the situation when the covariance function of the process is known, and, based on this, can be present a model of describing the process in state variables.

So, let there be the covariance matrix of the system output $\vec{y}(t)-\mathbf{K}_{y}(t, \tau)$. It is built on the basis of observing a random output process $\vec{y}(t)$ measured by a device with an observation matrix $\mathbf{C}(t)$ on the interval $T_{i} \leq t, \tau \leq T_{j}$. For the process $\vec{y}(t)$, under consideration, use its description in state variables (1) - (4) is used, for which it is necessary to find the matrices $\mathbf{A}(t), \mathbf{B}(t), \mathbf{C}(t)$, $\mathbf{Q}$ and $\mathbf{P}_{i}$, that is, to solve the problem of factorization covariance function factorization. The factorization problem can be solved in the time domain or in the frequency domain. To solve the factorization problem in the frequency domain, it is necessary to find the Fourier transform from (9). Since the only way to take into account the possible non-stationarity of the system's output process is factorization in the time domain, consider its capabilities. So, have

$$
\mathbf{K}_{y}(t, \tau)= \begin{cases}\mathbf{C}(t) \Theta(t, \tau) \mathbf{K}_{\mathrm{x}}(\tau, \tau) \mathbf{C}^{T}(\tau), & t \geq \tau \\ \mathbf{C}(t) \mathbf{K}_{x}(t, t) \Theta^{T}(\tau, t) \mathbf{C}^{T}(\tau), & \tau \geq t\end{cases}
$$

From the properties of transition matrices ${ }^{8,9,10}\left(\Theta\left(t, t_{0}\right)=\mathbf{A}(t) \Theta\left(t, t_{0}\right)\right)$, it can be concluded that the covariance function of the process $\vec{y}(t)$, generated by a system with an unknown structure, represented in the form of state variables, must have a separable (decomposed) form.

$$
\mathbf{K}_{y}(t, \tau)= \begin{cases}\mathbf{F}^{T}(t) \mathbf{G}(\tau), & t \geq \tau \\ \mathbf{G}^{T}(t) \mathbf{F}(\tau), & \tau \geq t\end{cases}
$$

where $\mathbf{F}^{T}(t)=\mathbf{C}(t) \Theta\left(t, t_{1}\right) ; \mathbf{G}(t)=\Theta\left(t_{1}, t\right) \mathbf{K}_{x}(t, t) \mathbf{C}^{T}(t) ; t_{1}-$ an arbitrary time variable in the domain of the process $\vec{y}(t)$ (take it equal to $T_{i}$ ).

\footnotetext{
${ }^{8}$ Van Trees, G.L. (1975). Teoriya obnaruzheniya, otsenok i modulyatsii [The theory of detection, estimation and modulation] (T. 2). Sovetskoye radio.

${ }^{9}$ Derusso, P., Roy, R., \& Klouz, C. (1970). Prostranstvo sostoyaniy $v$ teorii upravleniya [State space in control theory]. Nauka.

${ }^{10}$ Eikhoff, P. (1975). Osnovy identifikatsii sistem upravleniya [Fundamentals identification of control systems]. Mir.
} 
The dimensions $\mathbf{F}(t)$ and $\mathbf{G}(t)$ are related to the dimension of the output process $\vec{y}(t)$. Therefore, the first step in a factorization problem is to find the $(n \times m)$-dimensional matrices $\mathbf{F}(t)$ and $\mathbf{G}(t)$ by the given covariance functions. The second step is to develop an algorithm for obtaining $\mathbf{A}(t), \mathbf{B}(t), \mathbf{C}(t)$, $\mathbf{Q}$ and $\mathbf{P}_{i}$ from $\mathbf{F}(t)$ and $\mathbf{G}(t)$. Consider the case when no restriction is imposed on the minimality of $n$. In the case of non-minimal factorization, each element of the covariance matrix must have the form

$$
\left[\mathbf{K}_{y}(t, \tau)\right]_{i j}= \begin{cases}\sum_{k}^{n^{\prime}} \sum_{l}^{n^{\prime}} b_{i j}^{*}(k, l) f_{k}(t) q_{i}(\tau), & T_{i} \leq \tau \leq t \leq T_{j} \\ \sum_{k}^{n^{\prime}} \sum_{l}^{n^{\prime}} b_{i j}^{*}(k, l) q_{i}(t) f_{k}(\tau), & T_{i} \leq t \leq \tau \leq T_{j}\end{cases}
$$

The members of the series $\left\{f_{k}(t), 1 \leq k \leq n^{\prime}\right\},\left\{q_{i}(\tau), 1 \leq l \leq n^{\prime}\right\}$ will be, for example, for $n=2$, if

$$
\mathbf{K}_{y}(t, \tau)=\left[\begin{array}{cc}
\mathbf{P}_{1} e^{-k_{1}|t-\tau|} & 0 \\
0 & \mathbf{P}_{2} e^{-k_{2}|t-\tau|}
\end{array}\right],
$$

then

$$
\begin{array}{lll}
f_{1}(t)=e^{-k_{1} t} & q_{1}(\tau)=e^{k_{1} \tau} & b_{11}(k, l)=\mathbf{P}_{1} \delta_{k_{1}} \delta_{i_{1}} \\
f_{2}(t)=e^{-k_{2} t} & q_{2}(\tau)=e^{k_{2} \tau} & b_{22}(k, l)=\mathbf{P}_{2} \delta_{k_{2}} \delta_{i_{2}} \\
& & b_{12}(k, l)=b_{21}(k, l)=0
\end{array}
$$

In general, column vectors

$$
\vec{f}(t)=\left(\begin{array}{c}
f_{1}(t) \\
\vdots \\
f_{n}(t)
\end{array}\right), \quad \vec{q}(t)=\left(\begin{array}{c}
q_{1}(t) \\
\vdots \\
q_{n}(t)
\end{array}\right)
$$

form systems of linearly independent functions. Each element of matrix (12) can be written as:

$$
\left[\mathbf{K}_{y}(t, \tau)\right]_{i j}=\vec{f}^{T}(t)\left[\begin{array}{cccc}
g_{i j}^{*}(1,1) & g_{i j}^{*}(1,2) & \cdots & g_{i j}^{*}\left(1, n^{1}\right) \\
\vdots & & \ddots & \vdots \\
g_{i j}^{*}\left(n^{1}, 1\right) & & \cdots & g_{i j}^{*}\left(n^{1}, n^{1}\right)
\end{array}\right] \vec{q}(\tau)=\vec{f}^{T}(t) \mathbf{G}_{i j}^{*} \vec{q}(\tau),
$$

where $\mathbf{G}_{i j}^{*}-$ matrix of dimension $n^{1} \times n^{1}$. 
Using this representation, it is possible to decompose by factors, not necessarily of the minimum degree, the result is obtained in the form of matrices of dimension $\left(\left(n^{1} \cdot m\right) \times m\right)$ :

$$
\begin{gathered}
\mathbf{F}^{*}(t)=\left[\begin{array}{cccc}
\vec{f}(t) & 0 & \cdots & 0 \\
0 & \vec{f}(t) & \cdots & 0 \\
\vdots & \vdots & \ddots & \vdots \\
0 & 0 & \cdots & \vec{f}(t)
\end{array}\right], \\
\mathbf{G}^{*}(t)=\left[\begin{array}{cccc}
\mathbf{G}_{11}^{*} \vec{q}(t) & \mathbf{G}_{12}^{*} \vec{q}(t) & \cdots & \mathbf{G}_{1 n}^{*} \vec{q}(t) \\
\mathbf{G}_{21}^{*} \vec{q}(t) & \mathbf{G}_{22}^{*} \vec{q}(t) & & \\
\vdots & \vdots & \ddots & \vdots \\
\mathbf{G}_{m 1}^{*} \vec{q}(t) & 0 & \cdots & \mathbf{G}_{m n}^{*} \vec{q}(t)
\end{array}\right] .
\end{gathered}
$$

A sufficient criterion for verifying that the matrices $\mathbf{F}^{*}(t)$ and $\mathbf{G}^{*}(t)$ are factors of the minimum degree is the positive definiteness of two matrices

$$
\begin{gathered}
\mathbf{M}_{\mathbf{F}}=\int_{T_{i}}^{T_{j}} \mathbf{F}^{*}(t) \mathbf{F}^{* T}(t) d t, \\
\mathbf{M}_{\mathbf{G}}=\int_{T_{i}}^{T_{j}} \mathbf{G}^{*}(t) \mathbf{G}^{* T}(t) d t,
\end{gathered}
$$

that is, the rank of the matrices $\mathbf{M}_{\mathbf{F}}$ and $\mathbf{M}_{\mathbf{G}}$ is equal to the minimum dimension $\left(n^{*}=n^{1} \cdot m\right)$. Since $\mathbf{M}_{\mathbf{F}}$ and $\mathbf{M}_{\mathbf{G}}$ are symmetric, positively semi-definite, they must be coordinated through non-degenerate transformations $\mathbf{T}_{\mathbf{F}}$ and $\mathbf{T}_{\mathbf{G}}$ in accordance with matrices - conditional mathematical expectations $\mathrm{E}_{\mathrm{F}}$ and $\mathrm{E}_{\mathrm{G}}$. There is always a coordinate system in which random variables are uncorrelated $^{11}$, and the new system is associated with an output linear transformation, that is

$$
\begin{array}{lll}
\mathbf{T}_{\mathbf{F}} & \mathrm{E}_{\mathbf{F}} & \mathbf{T}_{\mathbf{F}}^{T}=\mathbf{M}_{\mathbf{F}}, \\
\mathbf{T}_{\mathbf{G}} & \mathrm{E}_{\mathbf{G}} & \mathbf{T}_{\mathbf{G}}^{T}=\mathbf{M}_{\mathbf{G}}
\end{array}
$$

and at this

${ }^{11}$ Bendat, J., \& Pirsol, A. (1989). Prikladnoy analiz sluchaynykh dannykh [Applied Analysis of Random Data]. Mir. 


$$
\begin{gathered}
\int_{T_{i}}^{T_{j}}\left(\mathbf{T}_{\mathbf{F}} \mathrm{E}_{\mathbf{F}} \mathbf{T}_{\mathbf{F}}^{-1} \mathbf{F}^{*}(t)-\mathbf{F}^{*}(t)\right)\left(\mathbf{T}_{\mathbf{F}} \mathrm{E}_{\mathbf{F}} \mathbf{T}_{\mathbf{F}}^{-1} \mathbf{F}^{*}(t)-\mathbf{F}^{*}(t)\right)^{T} d t=0, \\
\int_{T_{i}}^{T_{j}}\left(\mathbf{T}_{\mathbf{G}} \mathrm{E}_{\mathbf{G}} \quad \mathbf{T}_{\mathbf{G}}^{-1} \mathbf{G}^{*}(t)-\mathbf{G}^{*}(t)\right)\left(\mathbf{T}_{\mathbf{G}} \quad \mathrm{E}_{\mathbf{G}} \mathbf{T}_{\mathbf{G}}^{-1} \mathbf{G}^{*}(t)-\mathbf{G}^{*}(t)\right)^{T} d t=0 .
\end{gathered}
$$

As a result, get

$$
\begin{gathered}
\mathbf{F}^{*}(t)=\mathbf{T}_{\mathbf{F}} \mathrm{E}_{\mathbf{F}} \mathbf{T}_{\mathbf{F}}^{-1} \mathbf{F}^{*}(t), \quad T_{i} \leq t \leq T_{j} \\
\mathbf{G}^{*}(t)=\mathbf{T}_{\mathbf{G}} \mathrm{E}_{\mathbf{G}} \mathbf{T}_{\mathbf{G}}^{-1} \mathbf{G}^{*}(t), \quad T_{i} \leq t \leq T_{j} \\
\mathbf{K}_{\mathrm{y}}(t, \tau)=\mathbf{F}^{* T}(t) \mathbf{G}^{*}(\tau)=\mathbf{F}^{* T}(\mathrm{t}) \mathbf{T}_{\mathbf{F}}^{-1 T} \mathrm{E}_{\mathbf{F}} \mathbf{T}_{\mathbf{F}}^{T} \mathbf{T}_{\mathbf{G}} \mathrm{E}_{\mathbf{G}} \mathbf{T}_{\mathbf{G}}^{-1} \mathbf{G}^{*}(\tau), \quad t>\tau
\end{gathered}
$$

To obtain the minimum degree multiplier for (11), let $\mathbf{F}(t)$ and $\mathbf{G}(t)$ be defined as follows:

$$
\begin{aligned}
& \mathbf{F}(t)=\mathbf{N}_{1} \quad \mathbf{T}_{\mathbf{F}}^{-1} \mathbf{F}^{*}(t), \\
& \mathbf{G}(t)=\mathbf{N}_{2} \quad \mathbf{T}_{\mathbf{G}}^{-1} \mathbf{G}^{*}(t),
\end{aligned}
$$

where the matrices $\mathbf{N}_{1}$ and $\mathbf{N}_{2}$, of dimension $\left(n \times n^{*}\right)$, satisfy the condition $\mathrm{E}_{\mathbf{F}} \mathbf{T}_{\mathbf{F}}^{T} \mathbf{T}_{\mathbf{G}} \mathrm{E}_{\mathbf{G}}=\mathbf{N}_{1}^{T} \mathbf{N}_{2}$, and the matrix $\mathrm{E}_{\mathbf{F}} \mathbf{T}_{\mathbf{F}}^{T} \mathbf{T}_{\mathbf{G}} \mathrm{E}_{\mathbf{G}}$ has, respectively, the dimension $\left(n^{*} \times n^{*}\right)$. Now, having an algorithmic procedure for decomposition the covariance matrix $\mathbf{K}_{\mathrm{y}}(t, \tau)$ into factors, it is necessary to determine the state matrices of the dynamic system $\mathbf{A}(t), \mathbf{B}(t), \mathbf{C}(t)$ and the covariance matrices $\mathbf{Q}$ and $\mathbf{P}_{\mathrm{i}}$ on the basis of $\mathbf{F}(t)$ and $\mathbf{G}(t)$. Since the coordinate system of the state vector is not the only one and it follows from this that these matrices are deliberately not the only ones ${ }^{12,13}$.

Indeed, with the exception of its dimension, the matrix $\mathbf{A}(t)$ is essentially not set.

First, consider an implementation with a triplet of matrices $\left(0, \mathbf{B} *(t), \mathbf{C}_{*}(t)\right)$, and then - the question of the transformation to coordinate systems with the desired properties (here " 0 "' is the zero matrix $\mathbf{A} *(t)$ ). The transition matrix associated with $\mathbf{A} *(t)=0$ is the identity matrix ${ }^{14}$. Therefore, according to (10),

${ }^{12}$ Derusso, P., Roy, R., \& Klouz, C. (1970). Prostranstvo sostoyaniy v teorii upravleniya [State space in control theory]. Nauka.

${ }_{13}$ Gantmakher, F.R. (2004). Teoriya matrits [Matrix theory]. FIZMATLIT.

${ }^{14}$ Van Trees, G.L. (1975). Teoriya obnaruzheniya, otsenok $i$ modulyatsii [The theory of detection, estimation and modulation] (T. 2). Sovetskoye radio. 


$$
\mathbf{K}_{y}(t, \tau)=\left\{\begin{array}{l}
\mathbf{C}_{*}(t) \mathbf{K}_{x *}(\tau, \tau) \mathbf{C}_{*}^{T}(\tau), t>\tau \\
\mathbf{C}_{*}(t) \mathbf{K}_{x *}(t, t) \mathbf{C}_{*}^{T}(\tau), \tau>t
\end{array}\right.
$$

or

$$
\mathbf{F}^{T}(t)=\mathbf{C}_{*}(t), \quad \mathbf{G}(t)=\mathbf{K}_{x *}(t, t) \mathbf{C}_{*}^{T}(\tau)
$$

Note that (14) does not uniquely define $\mathbf{K}_{x *}(t, t)$, because the matrix $\mathbf{C} *(t)$ has the dimension $(m \times n)$, and the matrix $\mathbf{K}_{x^{*}}(t, t)$ has the dimension $(n \times n)$. Using (7), it is possible to show the validity of the following two properties

$$
\begin{gathered}
\dot{\mathbf{K}}_{*}(t, t)=\mathbf{B}_{*}(t) \mathbf{Q} \mathbf{B}_{*}(t), \\
\mathbf{F}^{T}(t) \dot{\mathbf{G}}(t)-\mathbf{G}^{T}(t) \dot{\mathbf{F}}(t)=\mathbf{C}_{*}(t) \mathbf{B}_{*}(t) \mathbf{Q} \mathbf{B}_{*}^{T}(t) \mathbf{C}_{*}(t) .
\end{gathered}
$$

It is known ${ }^{15}$ that the covariance function of the derivative of a random vector $\overrightarrow{\mathbf{y}}(t)$ is determined by the expression

$$
\mathbf{K}_{\mathbf{y}}(t, \tau)=\frac{\partial^{2}}{\partial t \partial \tau} \mathbf{K}_{y}(t, \tau)
$$

or

$$
\begin{aligned}
& \mathbf{K}_{y}(t, \tau)=\left\{\begin{array}{ll}
\dot{\mathbf{F}}^{T}(t) \dot{\mathbf{G}}(\tau), & t>\tau \\
\dot{\mathbf{G}}^{T}(t) \dot{\mathbf{F}}(\tau), & \tau>t
\end{array}\right\}+ \\
& +\left(\mathbf{F}^{T}(t) \dot{\mathbf{G}}(t)-\mathbf{G}^{T}(t) \dot{\mathbf{F}}(t)\right) \delta(t-\tau) .
\end{aligned}
$$

If the process is differentiable in the root mean square, then the coefficient at the $\delta$-function should be equal to zero ${ }^{16}$. Noting that $\mathbf{Q}$ can be considered positive definite without loss of generality, come to the conclusion that for a mean-square differentiable process

$$
\mathbf{C}_{*}(t) \mathbf{B}_{*}(t)=0 .
$$

\footnotetext{
${ }^{15}$ Bendat, J., \& Pirsol, A. (1989). Prikladnoy analiz sluchaynykh dannykh [Applied Analysis of Random Data]. Mir.

16 Ibid.
} 
For a differentiable process, the expansion, according to (18), has the form

$$
\begin{gathered}
\mathbf{F}_{\dot{\mathbf{y}}}^{T}(t)=\dot{\mathbf{F}}^{\mathrm{T}}(t)=\dot{\mathbf{C}}_{*}(t), \\
\mathbf{G}_{\mathbf{y}}(t)=\dot{\mathbf{G}}(t)=\mathbf{K}_{\mathrm{x} *}(t, t) \dot{\mathbf{C}}_{*}^{T}(\tau) .
\end{gathered}
$$

From this it follows that the implementation for the derivative of the process would be the function $(0, \mathbf{B} *(t), \mathbf{C} *(t))$. The inference strategy is to repeat this differentiation procedure until $\vec{y}^{(l)}(t)$ is reached. $\vec{y}^{(l)}(t)$ is the higher-order derivative of the process $\vec{y}(t)$, which still exists in the root-meansquare sense. The need for this procedure is that the decomposition of the process $\vec{y}(t)$ and all its derivatives up to and including $\vec{y}^{(l)}(t)$ is necessary to determine the state matrices. In the general case, for the interval $1 \leq k \leq l$ have

$$
\begin{gathered}
\mathbf{F}^{(k-1) T}(t) \mathbf{G}^{(k)}(t)-\mathbf{G}^{(k-1) T}(t) \mathbf{F}^{(k)}(t)=0, \\
\mathbf{C}_{*}^{(k-1)}(t) \mathbf{B}_{*}(t)=0 .
\end{gathered}
$$

The $l$-th order derivative has a realization $\left(0, \mathbf{B} *(t), \mathbf{C}_{*}^{(l)}(t)\right)$ and decomposition $\mathbf{C}_{*}^{(l)}(t)$ and $\mathbf{K}_{x^{*}}(t, t) \mathbf{C}_{*}^{(l)}(t)$ for $\mathbf{F}^{T}(t)$ and $\mathbf{G}(t)$, respectively.

Equations (19), (20), (21), (22) contain the main results connecting the differentiability of the process with the derivatives of the factors $\mathbf{F}(t)$ and $\mathbf{G}(t)$. The algorithm for determining the state matrices $\left(0, \mathrm{~B}^{*}(\mathrm{t}), \mathrm{C}^{*}(\mathrm{t})\right)$ can be reduced to the following sequence of operations ${ }^{17}$. Arrange the components $\mathbf{K}_{y}(t, \tau)$ in the reverse order of their differentiability, that is, the first $r_{1}$ components have only derivatives of zero order, the second $r_{2}$ components have only derivatives of first order, etc. It is also assumed that the columns of the matrices $\mathbf{F}(t)$ and $\mathbf{G}(t)$ are respectively interchanged and that $\mathbf{Q}$ is an $m$ - dimensional identity matrix. Next, partition the matrices $\mathbf{B} *(t)$ and $\mathbf{C}_{*}(t)$ in accordance with the order of differentiability $\left(r_{1}, r_{2}, \ldots, r_{L}\right)$ :

$$
\mathbf{B}_{*}(t)=\left[\begin{array}{llll}
\mathbf{B}_{*_{1}}(t) & \mathbf{B}_{*_{2}}(t) & \cdots & \mathbf{B}_{*_{L}}(t)
\end{array}\right]-n \text { components, }
$$

${ }^{17}$ Baggeroer, A.B. (1968). State Variables, the Fredholm Theory, and Optimal Communications. M.I.T. 


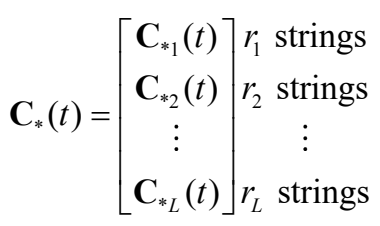

$n$ components

Each component $\vec{y}_{L}(t)$ has a realization $\left(0, \mathbf{B} *(t), \mathbf{C}_{* l}(\mathrm{t})\right)$, which is $(l-1)-$ fold (not $l$-fold) differentiable in the mean square sense (Fig. 1$)^{18}$.

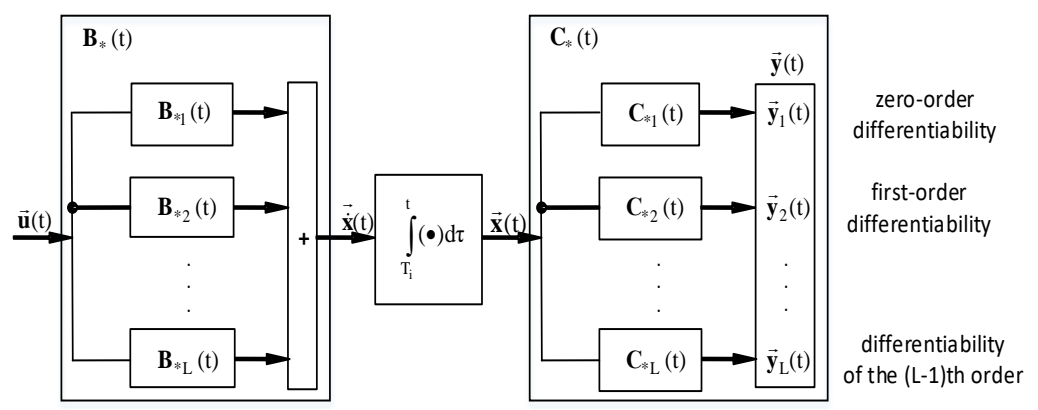

Fig. 1. Structural scheme of the implementation of the output process in accordance with the its components differentiability ${ }^{19}$

According to (15), the differential equation for the covariance function of the process is written in the form

$$
\dot{\mathbf{K}}_{*}(t, t)=\mathbf{B}_{*}(t) \mathbf{B}_{*}^{T}(t)=\sum_{l=1}^{L} \mathbf{B}_{* l}(t) \mathbf{B}_{* l}^{T}(t) .
$$

If (22) is applied to the corresponding components $\vec{y}_{i}(t)$, then it follows from the indicated differentiability conditions that

$$
\mathbf{C}_{*}^{(k)}(t) \mathbf{C}_{*}(t)=0 \text { при } 0 \leq k<l-1 .
$$

${ }^{18}$ Van Trees, G.L. (1972). Teoriya obnaruzheniya, otsenok $i$ modulyatsii [The theory of detection, estimation and modulation] (T. 1). Sovetskoye radio.

19 Ibid. 
Split the $\mathbf{F}(t)$ and $\mathbf{G}(t)$ in the form

$$
\begin{aligned}
& \mathbf{F}(t)=\left[\begin{array}{llll}
\mathbf{F}_{1}(t) & \mathbf{F}_{2}(t) & \cdots & \mathbf{F}_{L}(t)
\end{array}\right] \\
& \begin{array}{cccc}
r_{1} & r_{2} & \ldots & r_{L} \\
\text { columns } & \text { columns } & & \text { columns }
\end{array} \text {, } \\
& \mathbf{G}(t)=\left[\begin{array}{llll}
\mathbf{G}_{1}(t) & \mathbf{G}_{2}(t) & \cdots & \mathbf{G}_{L}(t)
\end{array}\right]
\end{aligned}
$$

Now, as it is obvious from (13), (14), it is necessary to identify $\mathbf{C} *(t)$ with $\mathbf{F}^{T}(t)$, that is,

$$
\begin{aligned}
& \mathbf{C}_{*}(t)=\mathbf{F}^{T}(t), \\
& \mathbf{C}_{* l}^{T}(t)=\mathbf{F}_{l}(t) .
\end{aligned}
$$

According to this, it is possible to freely mutually replace the matrices $\mathbf{F}(t)$ and $\mathbf{C}^{T}(t)$ in the subsequent equations. Thus

$$
\begin{aligned}
\mathbf{G}(t) & =\mathbf{K}_{x^{*}}(t, t) \mathbf{F}(t) \\
\mathbf{G}_{l}(t) & =\mathbf{K}_{x *}(t, t) \mathbf{F}_{l}(t)
\end{aligned}
$$

Differentiating (28), obtain

$$
\begin{aligned}
& \dot{\mathbf{G}}_{l}(t)=\dot{\mathbf{K}}_{*}(t, t) \mathbf{F}_{l}(t)+\mathbf{K}_{x *}(t, t) \dot{\mathbf{F}}_{l}(t)=\mathbf{B}_{*}(t) \mathbf{B}_{*}^{T}(t) \mathbf{C}_{* l}^{T}(t)+\mathbf{K}_{x *}(t, t) \dot{\mathbf{F}}_{l}(t) \\
& \text { If } l=1 \text {, then } \dot{\mathbf{G}}_{1}(t)=\mathbf{B}_{*}(t) \mathbf{B}_{*}^{T}(t) \mathbf{C}_{* 1}^{T}(t)+\mathbf{K}_{x *}(t, t) \dot{\mathbf{F}}_{l}(t) . \\
& \text { If } l \neq 1 \text {, then taking into account }(24) \quad \dot{\mathbf{G}}_{1}(t)=\mathbf{K}_{x *}(t, t) \dot{\mathbf{F}}_{l}(t) . \\
& \text { After } l \text {-fold differentiation }
\end{aligned}
$$

$$
\begin{gathered}
\dot{\mathbf{G}}_{l}^{(l)}(t)=\mathbf{B}_{*}(t) \mathbf{B}_{*}^{T}(t) \mathbf{C}_{* l}^{(l-1) T}(t)+\mathbf{K}_{x *}(t, t) \dot{\mathbf{F}}_{l}^{(l)}(t) \\
\mathbf{G}_{l}^{(k)}(t)=\mathbf{K}_{x *}(t, t) \mathbf{F}_{l}^{(k)}(t), \quad 0 \leq k \leq l-1
\end{gathered}
$$

Perform term-by-term multiplication (29) by $\mathbf{C}_{* l}^{(l-1)}(t)$, putting $\mathbf{C}_{* l}^{(l-1)}(t)$ in front, and using the result of permutation (30), obtain the matrix equation of dimension $\left(r_{l} \times r_{l}\right)$

$$
\left(\mathbf{C}_{* l}^{(l-1)}(t) \mathbf{B}_{*}(t)\right)\left(\mathbf{B}_{*}^{T}(t) \mathbf{C}_{* l}^{(l-1) T}(t)\right)=\mathbf{F}_{l}^{(l-1) T}(t) \mathbf{G}_{l}^{(l)}(t)-\mathbf{G}_{l}^{(l-1) T}(t) \dot{\mathbf{F}}_{l}^{(l)}(t)=\mathbf{D}_{l}(t),
$$

which defines the matrix system $\mathbf{D}_{l}(t)$. 
Suppose that the matrix $\mathbf{K}_{y}(t, \tau)$ is positive definite. It follows from this assumption and the differentiability conditions that $\mathbf{D}_{l}(t)$ is also a positive definite matrix; so it has a positive definite square $\operatorname{root}^{20}$. The matrix $\left(\mathbf{C}_{*_{l}}^{(l-1)}(t) \mathbf{B}_{*}(t)\right)$ of dimension $\left(r_{l} \times m\right)$ can be expressed as

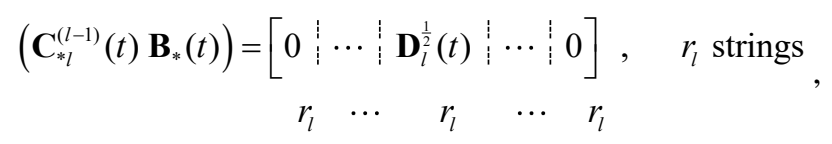

Now substitute the transposed matrix (31) into the formula (29)

$$
\mathbf{B}_{* l}(t)\left[\mathbf{D}_{l}^{\frac{1}{2}}(t)\right]^{T}=\mathbf{G}_{l}^{(l)}(t)-\mathbf{K}_{\mathrm{x} *}(t, t) \mathbf{F}_{l}^{(l)}(t),
$$

from here

$$
\mathbf{B}_{* l}(t)=\left(\mathbf{G}_{l}^{(l)}(t)-\mathbf{K}_{\mathrm{x} *}(t, t) \mathbf{F}_{l}^{(l)}(t)\right)\left[\mathbf{D}_{l}^{-\frac{1}{2}}(t)\right]^{T} .
$$

Equation (32) determines the decomposition $\mathbf{B} *(t)$ through $\mathbf{K}_{x^{*}}(t, t), \mathbf{F}(t)$ and $\mathbf{G}(t)$; however, $\mathbf{K}_{x^{*}}(t, t)$ remains unknown. Define the differential equation for $\mathbf{K}_{x^{*}}(t, t)$, substituting (32) into (23), obtain

$$
\begin{aligned}
& \dot{\mathbf{K}}_{*}(t, t)=\left\{\left[\begin{array}{llll}
\mathbf{G}_{1}^{(1)}(t) & \mathbf{G}_{2}^{(2)}(t) & \cdots & \mathbf{G}_{L}^{(L)}(t)
\end{array}\right]-\right. \\
& \left.-\mathbf{K}_{x *}(t, t)\left[\begin{array}{llll}
\mathbf{F}_{1}^{(1)}(t) & \mathbf{F}_{2}^{(2)}(t) & \cdots & \mathbf{F}_{L}^{(L)}(t)
\end{array}\right]\right\} \times \\
& \times\left[\begin{array}{cccc}
\mathbf{D}_{1}(t) & 0 & \cdots & 0 \\
0 & \mathbf{D}_{2}(t) & \cdots & 0 \\
\vdots & \vdots & \ddots & \vdots \\
0 & 0 & \cdots & \mathbf{D}_{L}(t)
\end{array}\right]^{-1}\left\{\left[\begin{array}{llll}
\mathbf{G}_{1}^{(1)}(t) & \mathbf{G}_{2}^{(2)}(t) & \cdots & \mathbf{G}_{L}^{(L)}(t)
\end{array}\right]-\right. \\
& \left.-\mathbf{K}_{x *}(t, t)\left[\begin{array}{llll}
\mathbf{F}_{1}^{(1)}(t) & \mathbf{F}_{2}^{(2)}(t) & \cdots & \mathbf{F}_{L}^{(L)}(t)
\end{array}\right]\right\}
\end{aligned}
$$

${ }^{20}$ Van der Waerden, B. L. (1976). Algebra [Algebra]. Nauka. 
This is a Riccati type differential equation ${ }^{21}$. In order to show the existence of a completely definite solution, it is necessary, using a number of substitutions, to reduce it to an ordinary differential equation, denoting

$$
\begin{aligned}
& {\left[\begin{array}{llll}
\mathbf{G}_{1}^{(1)}(t) & \mathbf{G}_{2}^{(2)}(t) & \cdots & \mathbf{G}_{L}^{(L)}(t)
\end{array}\right]=\tilde{\mathbf{G}}} \\
& {\left[\begin{array}{llll}
\mathbf{F}_{1}^{(1)}(t) & \mathbf{F}_{2}^{(2)}(t) & \cdots & \mathbf{F}_{L}^{(L)}(t)
\end{array}\right]=\tilde{\mathbf{F}}} \\
& {\left[\begin{array}{cccc}
\mathbf{D}_{1}(t) & 0 & \cdots & 0 \\
0 & \mathbf{D}_{2}(t) & \cdots & 0 \\
\vdots & \vdots & \ddots & \vdots \\
0 & 0 & \cdots & \mathbf{D}_{L}(t)
\end{array}\right]=\tilde{\mathbf{D}}}
\end{aligned}
$$

equation is obtained

$$
\dot{\mathbf{K}}_{*}(t, t)=\tilde{\mathbf{F}} \tilde{\mathbf{F}}^{\mathrm{T}} \mathbf{K}_{x *}^{2}(t, t)-\left(\tilde{\mathbf{D}} \tilde{\mathbf{G}} \tilde{\mathbf{F}}^{\mathrm{T}}+\tilde{\mathbf{D}} \tilde{\mathbf{F}} \tilde{\mathbf{G}}\right) \mathbf{K}_{x *}(t, t)+\tilde{\mathbf{D}} \tilde{\mathbf{G}}^{2}
$$

and introducing one more notation

$$
\begin{array}{rlrl}
\tilde{\mathbf{F}} \tilde{\mathbf{F}}^{\mathrm{T}} & =f(t) ; & & -\left(\tilde{\mathbf{D}} \tilde{\mathbf{G}} \tilde{\mathbf{F}}^{\mathrm{T}}+\tilde{\mathbf{D}} \tilde{\mathbf{F}} \tilde{\mathbf{G}}\right)=q(t) ; \\
\tilde{\mathbf{D}} \tilde{\mathbf{G}}^{2} & =h(t) ; & \mathbf{K}_{x *}(t, t)=z(t),
\end{array}
$$

obtain

$$
\dot{z}(t)=f(t) z^{2}(t)+q(t) z(t)+h(t)
$$

- the classical Riccati equation, which can be reduced by substituting $\phi(t)=\exp \left(-\int f z d t\right)$ into a nonzero solution of the linear differential equation

$$
f \phi^{\prime \prime}-\left(f^{\prime}+f q\right) \phi^{\prime}+f^{2} h \phi=0
$$

Conversely, since $f \neq 0$, then each nonzero solution of equation (36) by the transformation

\footnotetext{
${ }^{21}$ Wilkinson, J.H. (1972). Algebraicheskaya problema sobstvennykh znacheniy [Algebraic eigenvalue problem]. Nauka.
} 


$$
z(t)=\frac{\phi^{\prime}}{\phi f}
$$

is translated in a Riccati equation solution which, in turn, allows to estimate the state vector space $\vec{x}(t)$ and the matrices $\mathbf{A}(t), \mathbf{B}(t), \mathbf{C}(t)$. Denoting $f=a_{0}, \quad\left(f^{\prime}+f q\right)=a_{1}, \quad f^{2} h=a_{2}$, write the Laplace transform, equations (36), in the form

$$
\begin{aligned}
& \left(a_{0} s^{2}-a_{1} s+a_{2}\right) \phi=0 \\
& r_{1,2}=\frac{a_{1} \pm \sqrt{a_{1}^{2}-4 a_{0} a_{2}}}{2 a_{0}}
\end{aligned}
$$

The general solution of equation (36) $\phi=k_{1} e^{r_{1} t}+k_{2} e^{r_{2} t}$, and equation (35) according to (37) has the form

$$
Z=\mathbf{K}_{x *}(t, t)=\frac{k_{1} r_{1} e^{r_{1} t}+k_{2} r_{2} e^{r_{2} t}}{\left(k_{1} r_{1} e^{r_{1} t}+k_{2} r_{2} e^{r_{2} t}\right) \tilde{\mathbf{F}} \tilde{\mathbf{F}}^{T}}
$$

and, based on formulas (6), (25), (34)

$$
\begin{gathered}
\mathbf{K}_{y}(t, \tau)=\mathbf{C}(t) \mathbf{K}_{x}(t, \tau) \mathbf{C}^{T}(\tau)=\mathbf{C}_{*}(t) \frac{k_{1} r_{1} e^{r_{1} t}+k_{2} r_{2} e^{r_{2} t}}{\left(k_{1} e^{r_{1} t}+k_{2} e^{r_{2} t}\right) \tilde{\mathbf{F}} \tilde{\mathbf{F}}^{T}} \mathbf{C}_{*}^{T}(\tau)= \\
=\mathbf{F}^{T}(t) \frac{k_{1} r_{1} e^{r_{1} t}+k_{2} r_{2} e^{r_{2} t}}{\left(k_{1} e^{r_{1} t}+k_{2} e^{r_{2} t}\right) \tilde{\mathbf{F}} \tilde{\mathbf{F}}^{T}} \mathbf{F}(\tau)
\end{gathered}
$$

To determine the initial conditions for the solution of the Riccati equation, it suffices to require that the initial condition $\mathbf{K}_{x^{*}}\left(T_{i}, T_{j}\right)$ be described by a nonnegative definite symmetric matrix, $\mathbf{F}(t)$ and $\mathbf{G}(t)$ and their derivatives were finite and continuous, the covariance function was determined by relation (11) and at

$$
\begin{aligned}
\tilde{\mathbf{F}} & =\left[\begin{array}{llll}
\mathbf{F}_{1}^{(1)}(t) & \mathbf{F}_{2}^{(2)}(t) & \cdots & \mathbf{F}_{L}^{(L)}(t)
\end{array}\right] \\
\tilde{\mathbf{G}} & =\left[\begin{array}{llll}
\mathbf{G}_{1}^{(1)}(t) & \mathbf{G}_{2}^{(2)}(t) & \cdots & \mathbf{G}_{L}^{(L)}(t)
\end{array}\right]
\end{aligned}
$$

was a positive definite matrix. 
To determine the initial conditions, relation (30) can be expressed as a system of $L$ matrix equations of dimension $(n \times n)$ :

$$
\begin{aligned}
& {\left[\begin{array}{llll}
\mathbf{G}_{1}^{0}(t) & \mathbf{G}_{2}^{0}(t) & \cdots & \mathbf{G}_{L}^{0}(t)
\end{array}\right]=\mathbf{K}_{x *}(t, t)\left[\begin{array}{lllll}
\mathbf{F}_{1}^{0}(t) & \mathbf{F}_{2}^{0}(t) & \cdots & \mathbf{F}_{L}^{0}(t)
\end{array}\right]} \\
& {\left[\begin{array}{llll}
0 & \mathbf{G}_{2}^{1}(t) & \cdots & \mathbf{G}_{L}^{1}(t)
\end{array}\right]=\mathbf{K}_{x *}(t, t)\left[\begin{array}{llll}
0 & \mathbf{F}_{2}^{1}(t) & \cdots & \mathbf{F}_{L}^{1}(t)
\end{array}\right]} \\
& {\left[\begin{array}{llll}
0 & 0 & \cdots & \mathbf{G}_{L}^{L-1}(t)
\end{array}\right]=\mathbf{K}_{x *}(t, t)\left[\begin{array}{llll}
0 & 0 & \cdots & \mathbf{F}_{L}^{L-1}(t)
\end{array}\right]}
\end{aligned}
$$

It is necessary to solve these equations for the time moment $T_{i}$ and relatively $\mathbf{K}_{x^{*}}\left(T_{i}, T_{i}\right)$. It is possible in another way, using the method of pseudo-inversion of matrices to determine $\mathbf{K}_{x^{*}}\left(T_{i}, T_{i}\right)^{22}$, combining all equations (39) into an $(n \times n L)$-dimensional system. Equation (31) at the given initial conditions simulates the sought solution for $\mathbf{K}_{\mathrm{x} *}(t, t)$, which, in its turn, determines $\mathbf{B} *(t)$ through (32), and $\mathbf{C} *(t)$ is determined directly using $\mathbf{F}^{T}(t)$. Now there is a procedure for describing state variables. It is desirable to have an implementation of the solution of the system with constant parameters. Let's consider under what conditions this is possible. Let us introduce into consideration the matrix $\mathbf{T}(t)$, which determines the linear oneto-one differentiable transformation of the state vector $\vec{x}(t)$.

$$
\overrightarrow{\dot{x}}(t)=\mathbf{T}(t) \vec{x}(t)
$$

The state matrices for the transformed state vector are have the form:

$$
\begin{gathered}
\dot{\mathbf{A}}(t)=(\mathbf{T}(t) \mathbf{A}(t)+\dot{\mathbf{T}}(t)) \quad \mathbf{T}^{-1}(t) \\
\dot{\mathbf{B}}(t)=\mathbf{T}(t) \mathbf{B}(t) \\
\dot{\mathbf{C}}(t)=\mathbf{C}(t) \mathbf{T}^{-1}(t) \\
\dot{\mathbf{Q}}=\mathbf{Q} \\
\dot{\mathbf{P}}_{i}(t)=\mathbf{T}\left(T_{i}\right) \mathbf{P}_{i} \mathbf{T}^{T}\left(T_{i}\right)
\end{gathered}
$$

Let us determine the conditions under which it is possible to carry out such a transformation so that all state matrices are constant, that is, find conditions

${ }^{22}$ Gantmakher, F.R. (2004). Teoriya matrits [Matrix theory]. FIZMATLIT. 
when the matrices $\dot{\mathbf{A}}(t), \dot{\mathbf{B}}(t), \dot{\mathbf{C}}(t)$ are constant, and the initial matrices are

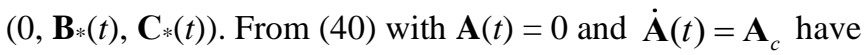

$$
\dot{\mathbf{T}}(t)=\mathbf{A}_{c} \mathbf{T}(t),
$$

where $\mathbf{A}_{c}$ - matrix to be determined.

The general solution to equation (45) has the form ${ }^{23}$

$$
\dot{\mathbf{T}}(t)=e^{\mathbf{A}_{c}\left(t-T_{i}\right)} \mathbf{T}\left(T_{i}\right)
$$

By $\dot{\mathbf{B}}(t)=\mathbf{B}_{c}$ and $\mathbf{B}(t)=\mathbf{B}_{*}(t)$, after substituting (46) into (41) and differentiating, the find that, to obtain a constant realization, it is necessary that the matrix $\mathbf{B} *(t)$ satisfies the equation

$$
\dot{\mathbf{B}}_{*}(t)=-\mathbf{B}_{*}(t)\left(\mathbf{T}^{-1}\left(T_{i}\right) \mathbf{A}_{c} \mathbf{T}\left(T_{\mathrm{i}}\right)\right) \mathbf{B}_{*}(t)=-\mathbf{A}_{\mathrm{T}} \mathbf{B}_{*}(t),
$$

where the matrix $\mathbf{A}_{\mathrm{T}}$ is defined explicitly. Similarly, $\mathbf{C} *(t)$ can be found as

$$
\dot{\mathbf{C}}_{*}(t)=\left(\mathbf{T}^{-1}\left(T_{i}\right) \mathbf{A}_{c} \mathbf{T}\left(T_{i}\right)\right)=\mathbf{C}_{*}(t) \mathbf{A}_{\mathrm{T}} .
$$

So for the existence of a realization of a system with constant (time independent) parameters, it is necessary and sufficient that there is a matrix $\mathbf{A}_{\mathrm{T}}$ that satisfies equations (47) and (48). Then there is a realization through the transformation of the general solution (46). The triplet of the implementation matrices will take the form $\left(\mathbf{T}\left(T_{i}\right) \mathbf{A}_{c} \mathbf{T}^{-1}\left(T_{i}\right), \mathbf{T}\left(T_{i}\right) e^{\mathbf{A}_{\mathrm{c}}\left(t-T_{i}\right)} \mathbf{B}_{*}(t), \mathbf{C}_{*}(t) e^{-\mathbf{A}_{c}\left(t-T_{i}\right)} \mathbf{T}^{-1}\left(T_{i}\right)\right){ }^{24}$.

Although equations (47) and (48) form a necessary and sufficient criterion for the existence of a realization with constant parameters, this criterion is rather difficult to use.

For the created method for identifying and predicting the state of dynamical systems in time, a method has been developed for determining the matrices of a

${ }^{23}$ Van der Waerden, B. L. (1976). Algebra [Algebra]. Nauka.

${ }^{24}$ Dymova, H.O. (2020). Metody i modeli uporyadkuvannya eksperymental'noyi informatsiyi dlya identyfikatsiyi i prohnozuvannya stanu bezperervnykh protsesiv [Methods and models for ordering experimental information for identifying and forecasting the state of continuous processes]. Knyzhkove vydavnytstvo PP Vyshemyrskyy V.S. 
dynamical system using the method of factorization covariance functions (solution of direct and inverse problems). The stages of the method for identifying and forecasting the state of dynamic systems are shown in Fig. 2.

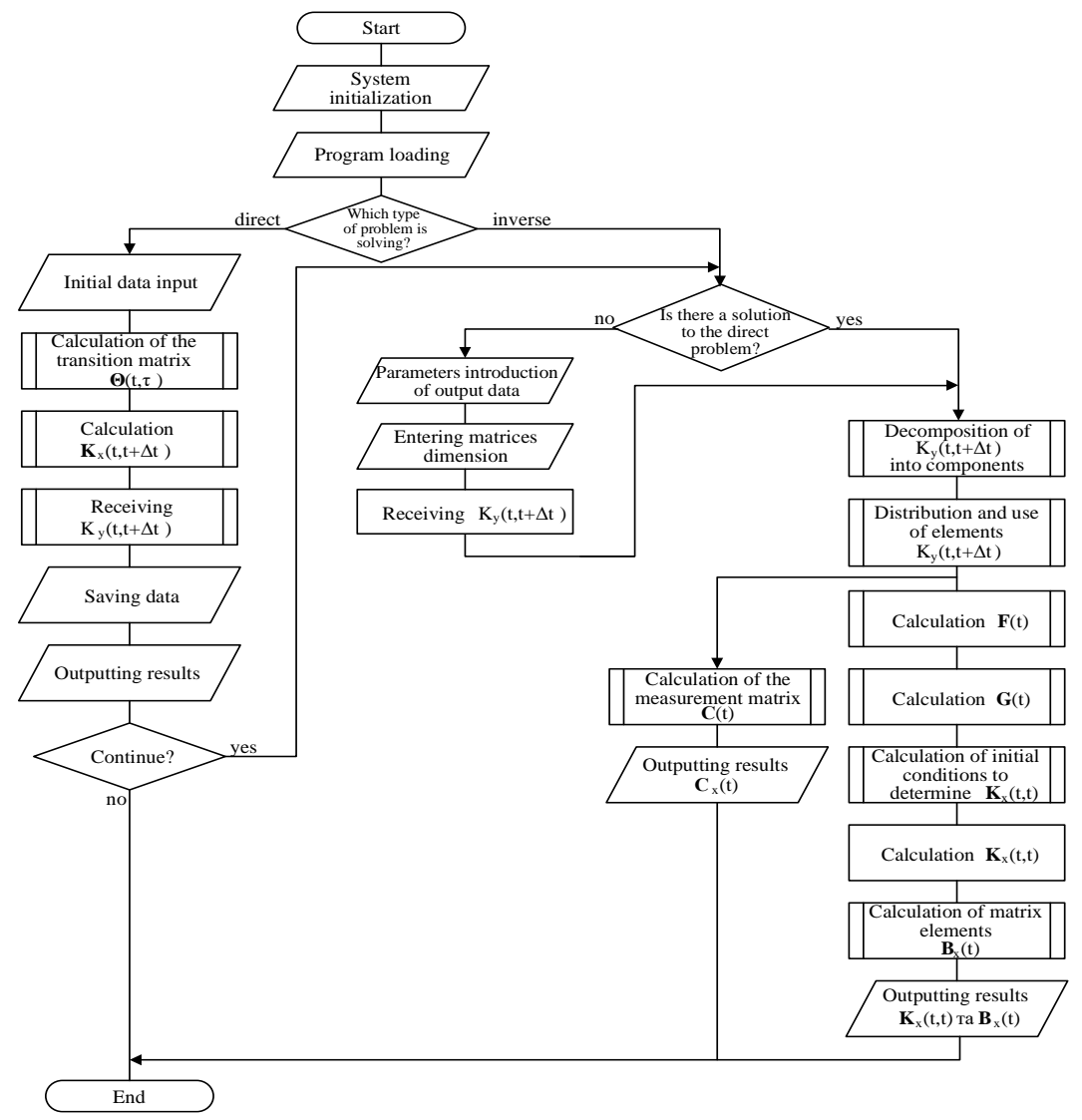

Fig. 2. Stages of the method for identifying and forecasting the state of dynamic systems

\section{CONCLUSIONS}

The work has solved the urgent scientific problem of identifying economic processes to determine the structure of a dynamic object with an output signal, the structure of its operator based on the structural properties of linear operators. A model and a method are developed that are used to identify incompletely defined dynamical systems. 
Methods for representing a random process in state variables and in the form of covariance functions are considered. The main difference between the method of specifying systems and random processes in state variables and the more common form of specifying them by the impulse transition function and the covariance function is that in the first case, the internal dynamics of the generating (modeling) system is determined, and not only the output process is described. It was shown how this internal form of the assignment can be used to define the characteristics of the output process. $n$ many problems, such a representation in state variables is explicitly present in the problem statement. Then the opposite situation arises, when the covariance function of the process is specified, and its generation requires performance in state variables. This problem is called the problem of factoring the covariance function into factors (or the problem of factorizing the covariance function).

The developed method for identifying and forecasting the state of dynamic systems with the improvement of the method of factorization of covariance functions, that is, the determination of the main matrices of a dynamic system using the Riccati equation. By the method of substitutions, this equation is reduced to a linear differential equation, the solution of which is transformed into a solution of the Riccati equation, with the help of the solution of which it is possible to determine the basic matrices of the dynamical system.

The use of the state space method allows us to reduce the problem of identifying the structure of a dynamical system using additional information about the random process it generates to solving the Riccati equation.

Based on one-to-one differential transformations of the state vector, the applied procedure makes it possible to reduce the problem of predicting the structure of a dynamic system to the problem of predicting the structure with constant matrices of the state space.

\section{SUMMARY}

There is a problem of identification, forecasting and data recovery in economic systems. The paper analyzes the problems of information recovery for dynamic systems. They are represented by three tasks: an identification task, a control task, and a measurement task. In these problems, only one of the components is unknown - either the characteristics of the system, or the output signal, or the input signal. In this paper, the task is set to determine the characteristics of the dynamic system and input data only from the output information. For this, a random process in state variables is presented and, according to its description, the covariance function of this process is determined. This procedure is a solution to the problem of forecasting the state of dynamic systems, that is, a solution to the direct problem, which is used to create signal libraries. To determine the partial identification of a dynamical system, a solution to the inverse problem was proposed using factorization 
method of covariance functions. There has been developed a method for identifying and forecasting the state of dynamical systems with the improvement of the method for factorizing covariance functions. The improvement of the method lies in the fact that the determination of the main matrices of the dynamical system was carried out using the Riccati equation.

\section{REFERENCES}

1. Baggeroer, A. B. (1968). State Variables, the Fredholm Theory, and Optimal Communications. M.I.T.

2. Bendat, J., \& Pirsol, A. (1989). Prikladnoy analiz sluchaynykh dannykh [Applied Analysis of Random Data]. Mir.

3. Derusso, P., Roy, R., \& Klouz, C. (1970). Prostranstvo sostoyaniy $v$ teorii upravleniya [State space in control theory]. Nauka.

4. Dymova, H. O. (2020). Metody $i$ modeli uporyadkuvannya eksperymental'noyi informatsiyi dlya identyfikatsiyi i prohnozuvannya stanu bezperervnykh protsesiv [Methods and models for ordering experimental information for identifying and forecasting the state of continuous processes]. Knyzhkove vydavnytstvo PP Vyshemyrs'kyy V.S.

5. Eikhoff, P. (1975). Osnovy identifikatsii sistem upravleniya [Fundamentals identification of control systems]. Mir.

6. Gantmakher, F. R. (2004). Teoriya matrits [Matrix theory]. FIZMATLIT.

7. Kamke, E. (1971). Spravochnik po obyknovennym differentsial'nym uravneniyam [Handbook of ordinary differential equations]. Nauka.

8. Van der Waerden, B. L. (1976). Algebra [Algebra]. Nauka.

9. Van Trees, G. L. (1972). Teoriya obnaruzheniya, otsenok $i$ modulyatsii [The theory of detection, estimation and modulation] (T. 1). Sovetskoye radio.

10. Van Trees, G. L. (1975). Teoriya obnaruzheniya, otsenok $i$ modulyatsii [The theory of detection, estimation and modulation] (T. 2). Sovetskoye radio.

11. Wilkinson, J. H. (1972). Algebraicheskaya problema sobstvennykh znacheniy [Algebraic eigenvalue problem]. Nauka.

\section{Information about the author: Dymova Hanna Olehivna,}

Phd.,Candidate of Technical Sciences, Associate Professor at the Department of Management and Information Technology

Kherson State Agrarian and Economic University 23, Stritenska str., Kherson, Ukraine, 73006 orcid.org/0000-0002-5294-1756 